\title{
MICRO-OPTIC SOLAR CONCENTRATION AND NEXT-GENERATION PROTOTYPES
}

\author{
Jason H. Karp, Eric J. Tremblay and Joseph E. Ford \\ Department of Electrical and Computer Engineering, University of California San Diego \\ 9500 Gilman Drive, La Jolla, CA 92093-0409, USA \\ jkarp@ucsd.edu
}

\begin{abstract}
We recently proposed a micro-optic solar concentrator using a two-dimensional array of small-aperture lenses focusing into a planar slab waveguide. By placing mirrors at each lens focus, light collected by the lens array reflects into a common slab waveguide at angles which guide by total internal reflection. Coupled sunlight propagates within the slab until reaching a photovoltaic cell mounted along the edge(s). Simulations of this geometry reveal designs with $89 \%$ and $81.9 \%$ optical efficiency at $100 x$ and $300 x$ geometric concentrations respectively. The microoptic concentrator was previously fabricated as a proof-ofconcept, but exhibited poor performance due to lens aberrations. Here, we present a $2^{\text {nd }}$-generation system using a better-suited lens array and achieve $>52 \%$ measured efficiency. We also discuss performance tradeoffs associated with micro-optic concentration and explore secondary coupler designs as a means to increase both efficiency and concentration.
\end{abstract}

\section{MICRO-OPTIC CONCENTRATION}

Concentrator photovoltaic (CPV) systems replace large photovoltaic (PV) active areas with optical components to collect and deposit solar energy onto small, efficient solar cells. Collection optics typically consist of primary lenses or mirrors focusing into secondary optical elements for flux homogenization prior to striking the PV cell [1]. A common approach reduces the system thickness by segmenting the large primary into an array of smaller diameter optical systems, each focusing onto individual PV cells and mounted onto a common tracking platform. Cell sizes and connectivity prevents this method from incorporating very small, millimeter-sized optics. If used, they could yield a substantially planar geometry and leverage volume manufacture such as roll-to-roll processing.

We propose an alternative approach which replaces individual secondary optics with a common slab waveguide and shared PV cell(s). Lenses mounted above the waveguide focus onto localized coupling regions which reflect sunlight at angles which guide by total internal reflection until reaching a PV cell typically located along the waveguide edge(s). Using a slab waveguide permits micro-optic primary lenses while homogenizing the output through divergence after coupling. Figure 1 compares individual concentrator modules with the described planar geometry.

The ratio of input to output apertures defines the geometric concentration ratio. For a rectangular micro-

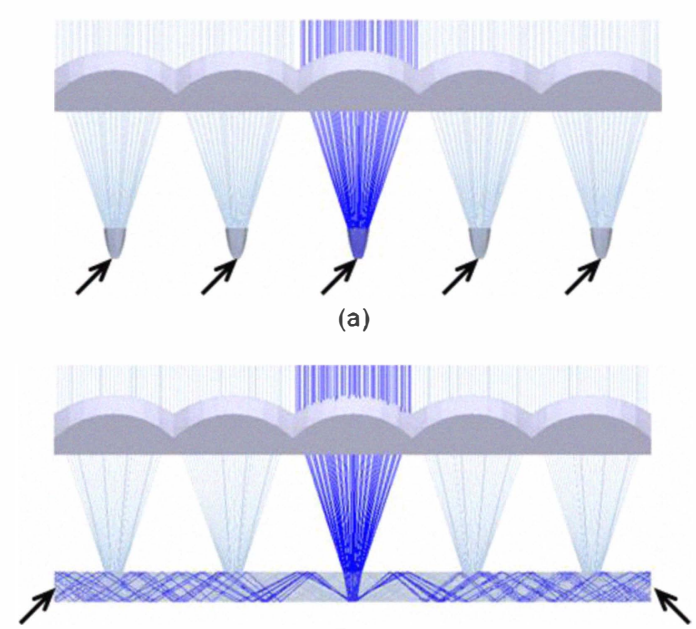

(b)

Fig. 1. Individual concentrators require multiple PV cells (a). Micro-optic concentration transports sunlight from all apertures to common PV cells (b). One ray path is highlighted in dark blue. Arrows indicate PV cell locations.

optic concentrator, this ratio is simply the waveguide length divided by two times the thickness, assuming symmetric coupling. The optical efficiency is the fraction of light which reaches the output and includes surface reflections, material absorption and waveguide propagation loss. Concentration in flux is the efficiency multiplied by the geometric concentration ratio. Planar waveguides, as opposed to tapered or wedged slabs, maintain the same modal cross-section and therefore permit guided rays to strike a subsequent coupling mirror and reflect out of the system as loss. Lens diameter, focal length to diameter ratio or F-number and aberrations influence the size and spacing of coupling regions and impact the loss per unit length within the waveguide.

To couple light into the waveguide, we selected reflective, $120^{\circ}$-apex symmetric prisms placed at each lens focus. Upon reflection, normal-incidence light tilts by $60^{\circ}$ which is exactly parallel to the adjacent facet and therefore avoids shadowing effects experienced by many other periodic structures. $120^{\circ}$ prisms couple light equally in opposing directions, creating output apertures along two waveguide edges.

We used ZEMAX non-sequential ray-tracing to model and optimize the performance of the geometry. Analyses used weighted AM1.5 sunlight from 0.4 to $1.6 \mu \mathrm{m}$ at $\pm 0.26^{\circ}$ field angles and modeled lens aberrations, surface reflections, 
dispersion and material absorption. A simulated $\mathrm{Mg}_{2} \mathrm{~F}_{2}$ antireflection coating was used on the lens array surface.

One optimized design used $2.38 \mathrm{~mm}$ diameter, $F / 2.45$ glass lenses $\left(B K 7, n_{d}=1.517\right)$ focusing onto $78 \mu \mathrm{m}$ coupling regions attached to a $1 \mathrm{~mm}$ thick glass waveguide ( $F 2$, $\mathrm{n}_{\mathrm{d}}=1.620$ ) surrounded by air as a cladding. At $100 \mathrm{x}$ geometric concentration, the system reached $89 \%$ optical efficiency and $81.9 \%$ at $300 x$ [2]. The glass components exhibited excellent spectral performance with no observable absorption peaks over the simulated waveguides. Absorption and decoupling losses scale with the optical path length within the waveguide leading to a reduction in efficiency at higher concentrations. These initial results were very encouraging and prompted an experimental proof-of-concept.

\section{EXPERIMENTAL PROTOTYPES}

Incorporating thousands of micro-optic lenses, all coupling into a common slab waveguide creates demanding lateral and rotational alignment tolerances between the two components. For example, the optimized design requires $<10 \mu \mathrm{m}$ lateral alignment and $<0.01^{\circ}$ rotational accuracy over $600 \mathrm{~mm}$ (300x concentration). To avoid difficult and expensive assembly, we pursue a self-aligned fabrication technique.

\section{Self-aligned fabrication}

As a means to fabricate coupling prisms on the surface of the waveguide, we use optical lithography wherein the prism facets are molded from SU-8 photopolymer. The lens array is fixed on the opposing side and a UV dosage through the array induces cross-linking at each focal region. Chemical development of the uncured polymer leaves appropriately sized and positioned coupling facets as part of the final device without requiring precision alignment. Controlling the angular divergence during the UV exposure determines the diameter of the formed coupling regions. A detailed description of the fabrication process can be found in Ref [3]. Self-aligned fabrication influenced the choice of $120^{\circ}$ symmetric prisms, which couple light regardless of incidence location along the prism period.

\section{$1^{\text {st }}$-Generation proof-of-concept}

We constructed a $1^{\text {st }}$-generation proof-of-concept to demonstrate self-aligned fabrication and waveguide coupling. The system used an inexpensive, commercially available $2.29 \mathrm{~mm}$ diameter, $F / 1.1$ acrylic lens array. A $75 \mathrm{~mm}$ long, $1 \mathrm{~mm}$ thick BK7 glass waveguide yielded $37.5 \mathrm{x}$ geometric concentration when used with the symmetric coupling prisms. The lens array formed $200 \mu \mathrm{m}$ coupling spots when illuminated with $\pm 0.26^{\circ}$ collimated light. SU-8 photoresist spun onto the waveguide surface was molded using a $50 \mu \mathrm{m}$ pitch, $15 \mu \mathrm{m}$ deep $120^{\circ}$ prism master.
Inputting the real component characteristics into our ZEMAX model showed the system could at best provide $44.8 \%$ optical efficiency. The poor predicted efficiency is mostly caused by the low F-number lens array which had strong spherical aberration, axial color and reduced array fill-factor between apertures. The optical efficiency was experimentally measured at $32.4 \%$ using a calibrated photodiode attached to the waveguide edge. $\pm 1.0^{\circ}$ angular acceptance was observed at $90 \%$ of the maximum. Images of the fabricated couplers and the complete $1^{\text {st }}$-generation system are shown in Fig. 2. A $50 \mu \mathrm{m}$ annulus of partially-cured photoresist surrounds each coupler and indicates aberration caused by the extreme lens curvature. Additional experimental losses are attributed to small fabrication imperfections and scattering at the lens and waveguide surfaces.

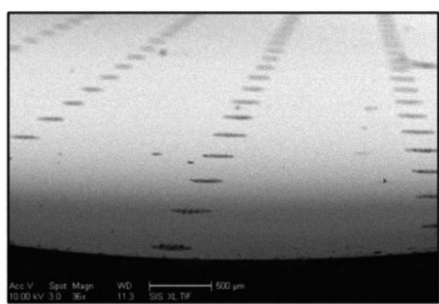

(a)

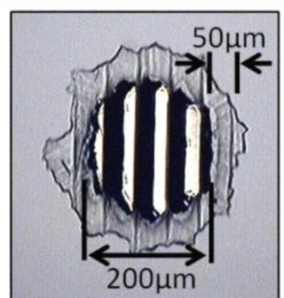

(b)

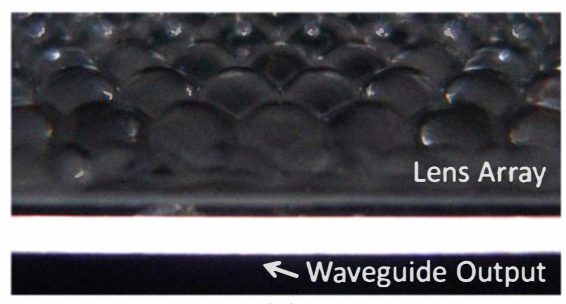

(c)

Fig. 2. SEM image of $120^{\circ}$ symmetric prism couplers fabricated on the back waveguide surface (a). Self-aligned lithography through F/1.1 lenses created 200 $\mathrm{mm}$ coupling regions (b). The $1^{\text {st }}$-generation prototype coupled $32.4 \%$ of incident light to the waveguide edges (c).

\section{$2^{\text {nd }}$-Generation prototype}

For a $2^{\text {nd }}$-generation prototype, we selected a $1.0 \mathrm{~mm}$ pitch, F/3.01 lens array. These acrylic optics were near diffraction-limited on-axis and focus to $40 \mu \mathrm{m}$ spots with $\pm 0.26^{\circ}$ field angles. Reduced lens sag also removed gaps between apertures to improve the overall array fill-factor. We simulated the performance of the $2^{\text {nd }}$-generation design and compared the optical efficiency to the optimized and $1^{\text {st }}$-generation systems, Fig. 3 .

We calculated $74.7 \%$ and $68.4 \%$ optical efficiencies at $100 x$ and $300 x$, respectively for the $2^{\text {nd }}$-generation system. Simulations show that $75 \mathrm{~mm} \times 1 \mathrm{~mm}$ waveguides $(37.5 \times$ concentration), are capable of reaching $76.2 \%$ efficiency. 

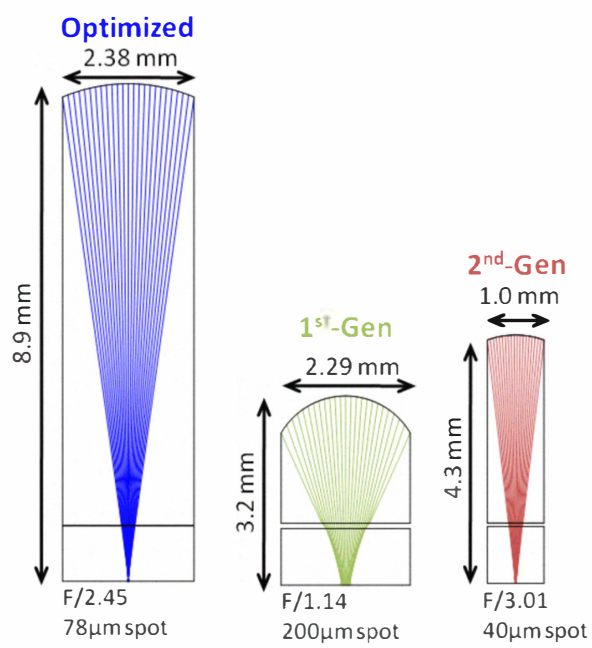

(a)

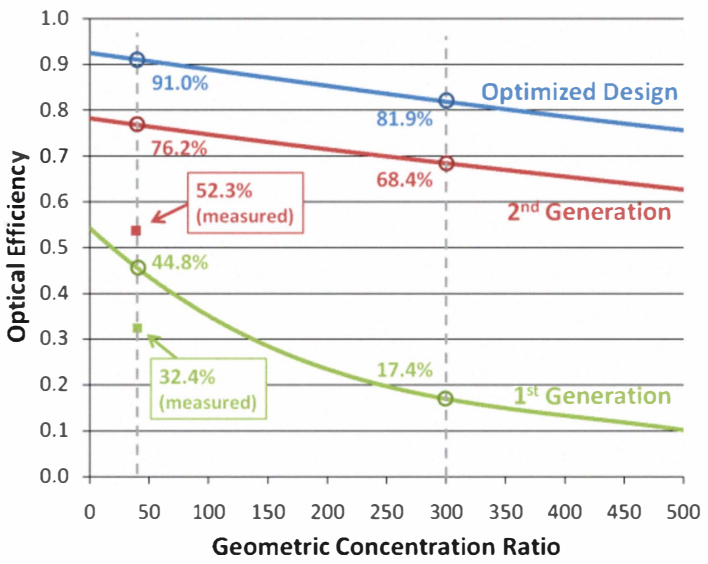

(b)

Fig. 3. Layouts for the three micro-optic concentrator designs: optimized, $1^{\text {st }}$-generation and $2^{\text {nd }}$-generation systems (a). Optical efficiencies for each system are plotted as functions of geometric concentration ratio (b).

Though the diameters of the new lenses are smaller than those of the optimized design, the slope of the concentration versus efficiency curves are quite comparable, meaning both systems experience similar absorption and decoupling losses. The primary difference occurs from an initial fixed loss due to the exclusion of an antireflection coating.

We fabricated the $2^{\text {nd }}$-generation system using the same molding and self-aligned exposure technique previously described. Images of the fabricated couplers are shown in Fig. 4. The SEM image shows a $41 \mu \mathrm{m}$ coupler with near vertical sidewalls. The new lens array reduces spherical aberration and therefore almost completely eliminates the annulus of partially cured photoresist.

The device was tested in the laboratory using a Xe arc lamp collimated to $\pm 0.26^{\circ}$ to represent the spectrum of the sun. To collect the output, a $30 \mathrm{~mm} \times 3 \mathrm{~mm}$ Hamamatsu photodiode (S3588) was positioned to span $25 \mathrm{~mm}$ of the $50 \mathrm{~mm}$ exit aperture on one side of the waveguide. We moved the detector to two locations along each output and experimentally measured $52.3 \%$ optical efficiency with $\pm 0.38^{\circ}$ angular acceptance. This measurement is below the simulated efficiency of $76.2 \%$, however, it does show a significant improvement over the initial proof-of-concept.

We have identified several loss mechanisms which reduce the overall optical efficiency. First, couplers with vertical sidewalls and diameters comparable to the prism pitch cause light reflected near the facet edge to leak through the sidewall and not couple into the waveguide. The complete solution reduces the prism array pitch so focused sunlight strikes multiple facets. To proceed without new components, we have adjusted the UV illumination to create oversized coupling regions $(\sim 100 \mu \mathrm{m})$ and provide additional prism periods at the cost of propagation loss. Other losses are attributed to the sputtered aluminum coating applied at the coupling surface. Coating reflectivity ranged between $83-87 \%$, falling short of the expected $92 \%$. Depositing highly reflective aluminum is a known issue and can be improved by optimizing process parameters [5]. Additionally, the metal can be seen peeling away at the coupler, further reducing the usable facet area and suggesting insufficient surface adhesion. We are currently exploring process adjustments and other coating materials to increase both reflectivity and adhesion.

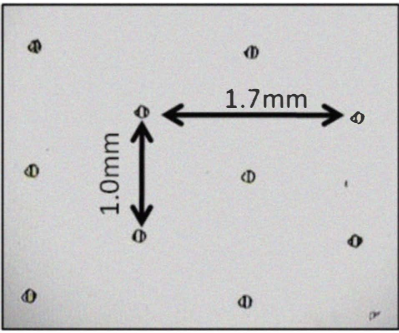

(a)

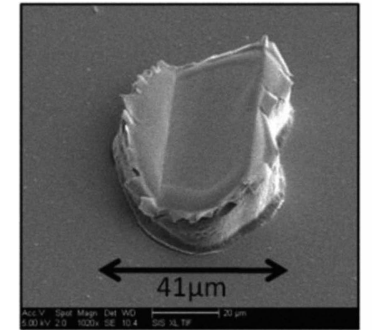

(b)

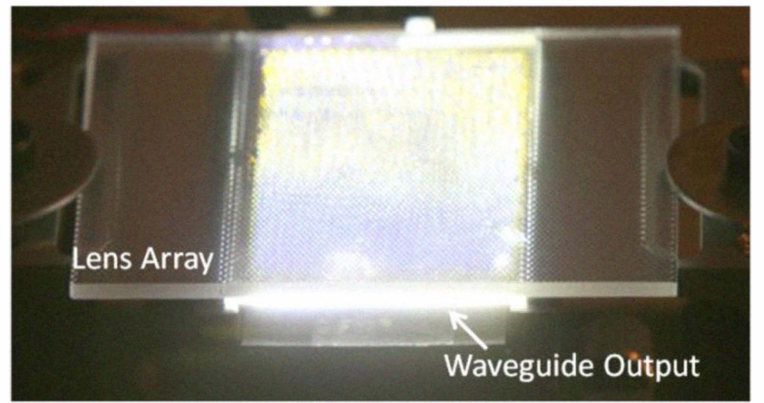

(c)

Fig. 4. Through self-alignment, the F/3.01 lens array created couplers as small as $41 \mu \mathrm{m}$ couplers, spaced $1.0 \mathrm{~mm}$ apart $(a, b)$. This $2^{\text {nd }}$-generation system demonstrated $52.3 \%$ optical efficiency (c). 


\section{SECONDARY CONCENTRATION}

Étendue, a property of all optical systems, states the lens aperture multiplied by the source angle remains constant [4]. To increase geometric concentration (by reducing the output area), we must increase the angular extent of the exiting light. Thermodynamics sets an upper bound which occurs when the output angles span $\pm 90^{\circ}$. In its current description, the micro-optic geometry relies solely on the lens array as the concentrating element. Planar waveguides do not alter coupled ray angles and therefore cannot contribute to further concentration.

The lens F-number specifies the cone of light coupled into the waveguide. Plano-convex lenses suffer from severe spherical aberration below $\sim F / 2$ and fall well short of the thermodynamic limit. Secondary concentration places an additional optical element at the waveguide exit aperture to remap the ray angles and increase the overall flux.

Light exiting the waveguide is confined within two ray bundles defined by the lens F-number, biased at $\pm 30^{\circ}$ after coupling. Fig. 5a shows a free-form reflector sitting between two opposing micro-optic concentrators. This secondary concentrator remaps the ray angles from both systems to further fill the cone of light striking the PV cell. As a result, the optic focuses to an area 3.6x smaller than the combined waveguide outputs. This configuration has the practical benefit of orienting the PV cell beneath the waveguide, providing space for the cell carrier, heatsink and electrical contacts. Fig. 5b plots the normalized intensity as a function of angle space while maintaining the same total power (area under the curve).

Absorption and decoupling losses both scale with optical path length to the exit aperture. Lower concentration systems provide very high optical efficiency, but do not reach the high flux levels required by current multi-junction solar cells. To maintain high efficiency and high concentration ratios, we combine secondary concentration with medium concentration micro-optics. For a 300x system incorporating $3.6 \mathrm{x}$ secondary concentration, we utilize two, $83 \times$ micro-optic concentrators, each with $89.5 \%$ optical efficiencies. Assuming a $98 \%$ reflective silver coating on the secondary optic, this configuration would achieve $87.7 \%$ efficiency compared to $81.9 \%$ when using only a lens array and waveguide. Fig. $5 c$ shows the described configuration using secondary optics. A simple mirror placed along one edge provides a single-output waveguide.

Another approach to secondary concentration orients coupling prisms to reflect light towards a limited region along the waveguide width. This method of orthogonal concentration provides up to $5 x$ concentration with $20 \%$ less propagation loss [6]. Radial coupling may be used in conjunction with secondary concentration as a means to achieve very high concentration while maintaining high optical efficiency.

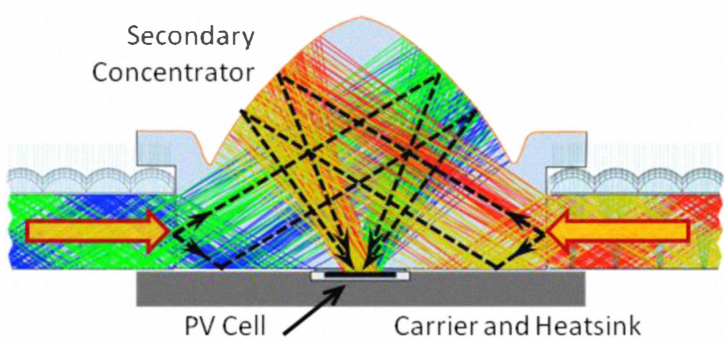

(a)

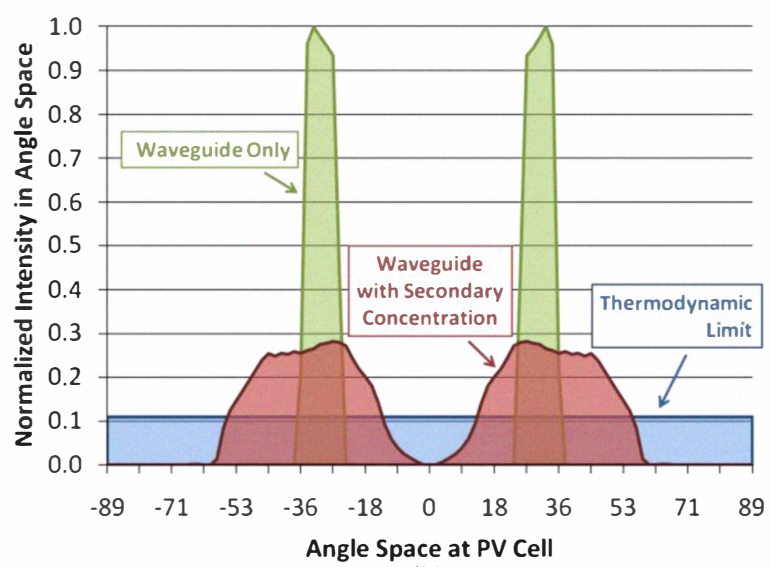

(b)

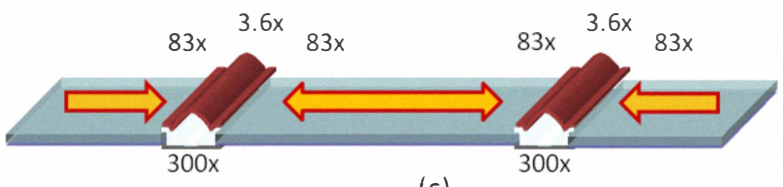

(c)

Fig. 5. A secondary optic focuses light from opposing waveguides with $3.6 \mathrm{x}$ additional concentration (a). The optic increases the angular extent of rays reaching the PV cell (b). Medium-concentration micro-optics paired with secondary concentration can increase overall efficiency (c).

\section{CONCLUSION}

Micro-optic solar concentration replaces individual optics with a two-dimensional lens array and common slab waveguide. Fabricating reflective couplers at each lens focus redirects incident sunlight into the waveguide and transports the energy to a PV cell along the edge. We fabricated a $1^{\text {st }}$-generaton proof-of-concept using a selfaligned lithography technique where the lens array focus was used as a mask to create each coupling region. We constructed a $2^{\text {nd }}$-generation prototype using a new lens array and experimentally demonstrated $52.3 \%$ optical efficiency. Secondary optics placed at the waveguide edge can increase the angular extent of the guided rays to provide an additional concentration factor. Combining secondary concentration with medium-concentration micro-optics provides a means to increase concentration while minimizing propagation loss. 


\section{ACKNOWLEDGEMENTS}

This research is supported by the National Science Foundation SGER (ECCS-0844274) and the California Energy Commission EISG (55780A/08-04).

\section{REFERENCES}

[1] J. M. Gordon, "Concentrator Optics," in Concentrator Photovoltaics, A. L. Luque and V. M. Andreev, (Springer, Berlin, 2007).

[2] J. H. Karp, E. J. Tremblay and J. E. Ford, "Planar micro-optic solar concentrator," Optics Express, Vol. 18, Issue 2, 11221133 (2010).

[3] J. H. Karp and J. E. Ford, "Planar micro-optic concentration using multiple imaging lenses into a common slab waveguide," Proc. SPIE 7407, 7407-11 (2009).

[4] R. Winston, J. C. Minano, W. T. Welford, and P. Benitez, Nonimaging Optics, (Academic Press 2004).

[5] S. Van Gils, Th. Dimogerontakis, G. Buytaert, E. Stijns, H. Terryn, P. Skeldon, G. E. Thompson, and M. R. Alexander, "Optical properties of magnetron-sputtered and rolled aluminum," J. Appl. Phys. 98, 083505 (2005).

[6] J. Karp, E. Tremblay, and J. Ford, "Radial Coupling Method for Orthogonal Concentration within Planar Micro-Optic Solar Collectors," Optics for Solar Energy, OSA, paper STuD2 (2010). 